M. Herrmann, J. Ribbe and N. V. Trung

Nagoya Math. J.

Vol. 124 (1991), 1-12

\title{
REES ALGEBRAS OF NON-SINGULAR EQUIMULTIPLE PRIME IDEALS*
}

\author{
M. HERRMANN, J. RIBBE AND N.V. TRUNG
}

\section{Dedicated to Professor H. Matsumura on occasion of his 60 th birthday}

\section{\$1. Introduction}

In a recent paper [HI2] the first named author and S. Ikeda have investigated Gorenstein properties under blowing up of height two equimultiple ideals from the arithmetical point of view. The problem is to what extent does the Gorenstein property of the Rees algebra $R(a)=$ $\oplus_{n \geq 0} \mathfrak{a}^{n}$ of an equimultiple ideal $\mathfrak{a}$ of a local ring $(A, \ldots)$ describe the structure of $A$ and a. One result is that if $A$ is a generalized CohenMacaulay ring (i.e. a ring of finite local cohomology) with $\operatorname{dim}(A) \geqq 4$ and if there is an height two equimultiple prime ideal $p$ such that $R(p)$ is Gorenstein, then $A$ is Gorenstein and $p$ is generated by a regular sequence (see [HI2], Theorem 2.6). That has led to the question whether this result is still valid for $\operatorname{dim}(A)=3$. $\Lambda$ partial answer was griven in [HL2], Proposition 2.10, where "equimultiplicity" was replaced by the stronger condition " $p / p^{2}$ is flat over $A / p$ ".

In this paper we extend first Theorem 2.6 in [HI2] to the CohenMacaulay case in the following sense, assuming that $\# A / m=\infty$.

Theorem (1.1). Let $(A, m)$ be a generalized Cohen-Macaulay ring with $\operatorname{dim}(A) \geqq 4$. Assume that there is a height two equimultiple prime ideal p) of $A$ such that

(i) $A_{\mathfrak{p}}$ is regular

(ii) $R(\mathfrak{p})$ is Cohen-Macaulay.

Then $A$ is a Cohen-Macaulay ring and $p$ is generated by a regular sequence.

Since $A_{p}$ is regular if $R(p)$ is Gorenstein [I], we obtain Theorem 2.6

Received October 16, 1989.

$\therefore$ We thank the referce for his detailed suggestions and improvements. 
of [HI2] from the proof of Theorem (1.1) without using the existence of dualizing complexes.

Note that if $\operatorname{dim}(A)=3$, the existence of such an ideal $\mathfrak{p}$ will already imply that $A$ is generalized Cohen-Macaulay, see [HI2], Theorem 3.1. Therefore it seems reasonable to conjecture that Theorem (1.1) also holds for the case $\operatorname{dim}(A)=3$; see [HI2], Remark 3.2. We shall see that this conjecture does not hold in general, (Example (6.2)). For that purpose we will develop a criterion for the Cohen-Macaulayness of $R(\mathfrak{p})$ for almost complete intersections $\mathfrak{p}$ in 3 -dimensional rings $A$. This criterion gives an explicit description of the presentation of the graded ring $\operatorname{gr}_{A}(\mathfrak{p})$ which depends only on the factor ring $A / \mathfrak{p}$.

Theorem (1.2). Let $(A, \mathfrak{m})$ be a local ring with $\operatorname{dim}(A)=3$ and $\frac{1}{2} \in A$. Let $\mathfrak{p}$ be a height two equimultiple ideal in $A$ such that $\mu(\mathfrak{p})=3$ and $A_{\mathfrak{p}}$ is regular. Then the following conditions are equivalent:

(1) $R(\mathfrak{p})$ is Cohen-Macaulay

(2) The associated graded ring has a presentation

$$
\operatorname{gr}_{A}(\mathfrak{p}) \cong K[X, Y, Z] /(f): a^{2},
$$

where $X, Y, Z$ are indeterminates over $K:=A / \mathfrak{p}$, and $f=a X+b Y+c Z \epsilon$ $K[X, Y, Z]$ such that $a \neq 0, b, c \in K$ and

(i) $b^{2}, c^{2}, b c \in a^{2} K$

(ii) $a K: b=a K: c=(a, c) K: b$.

Remark. The notation $(f): a^{2}$ will always mean $f \cdot K[X, Y, Z]: a^{2}$ in the sequel.

\section{§2. Preliminaries}

We shall see that in dealing with problems on Cohen-Macaulay Rees algebras, one can restrict the investigation to the associated graded rings which are easier to be handled-due to the special graded structure. We mention two results which are used frequently in the following sections. $(A, \mathfrak{m})$ is a local ring with $\operatorname{dim}(A)=d$ and $\mathfrak{a}$ an ideal of $A$ such that $\mathfrak{a} \not \sqrt{0_{A}}$. Let $R(\mathfrak{a})$ be the Rees algebra of $\mathfrak{a}, G$ the associated graded ring $\operatorname{gr}_{A}(\mathfrak{a})$ and $\mathfrak{M}$ the maximal homogeneous ideal of $G$. Then (see also [HI0], Theorem (4.4.1)) we have

Theorem (2.1) [TI, Theorem 1.1]. $R(\mathfrak{a})$ is Cohen-Macaulay if and only if 
(1) $\left[H_{\mathfrak{m}}^{i}(G)\right]_{n}=0$ for $n \neq-1, i=0, \cdots, d-1$

(2) $\left[H_{\mathfrak{m}}^{d}(G)\right]_{n}=0$ for all $n \geqq 0$.

In this case $\left[H_{\mathfrak{m}}^{i}(G)_{-1}=H_{\mathfrak{M}}^{i}(A)\right.$ for $i=0, \cdots, d-1$.

Proposition (2.2) [HI2, Proposition 2.1]. Let $d \geqq 3$. If there exists an equimultiple prime ideal $\mathfrak{p} \subset A$ of height 2 such that $R(\mathfrak{p})$ is CohenMacaulay and $A_{\mathfrak{p}}$ is regular, then $A$ and $G$ are domains satisfying Serre's condition $S_{2}$.

\section{§3. Proof of Theorem (1.1)}

From the assumption (ii) of the theorem we get by [HIO], Proposition (45.4) that

$$
\operatorname{depth}(A) \geqq \operatorname{dim}(A / \mathfrak{p})+1 \geqq 3 .
$$

Let $(x, y)$ be a minimal reduction of $\mathfrak{p}$. Since $A$ is a generalized Cohen-Macaulay ring which satisfies Serre's conditions $S_{2}$, the elements $x, y$ form an $A$-regular sequence. Moreover $(x, y) A$ has no embedded components in this case because of $\operatorname{depth}(A) \geqq 3$, and further $\mathfrak{p} A_{\mathfrak{p}}=(x, y) A_{\mathfrak{p}}$ by condition (i). Hence $\mathfrak{p}=(x, y) A$. Consequently

$$
R(\mathfrak{p})=A[X, Y] /(x Y-y X),
$$

where $X, Y$ are indeterminates over $A$. Therefore $A$ is a Cohen-Macaulay ring since $x Y-y X$ is a nonzerodivisor in $A[X, Y]$. For the same reason $A$ is Gorenstein if $R(p)$ is so.

Remark. If $\operatorname{dim}(A)=3$ then by [HI2], Theorem 3.1:

$$
\mathfrak{p} /(x, y) A \cong H_{\mathrm{m}}^{2}(A),
$$

where $H_{\mathrm{m}}^{i}(A)$ denotes the local cohomology of $A$ with respect to $\mathrm{m}$. Moreover, $H_{\mathrm{m}}^{0}(A)=H_{\mathrm{m}}^{1}(A)=0$, because $A$ satisfies $S_{2}$. Hence $A$ is CohenMacaulay if and only if $\mathfrak{p}=(x, y) A$. But $H_{\mathrm{m}}^{2}(A)$ needs not to be zero as we will see in the example (6.2).

\section{§4. Proof of Theorem (2.1), (1) $\Rightarrow(2)$}

Under the general assumptions of Theorem (1.2), condition (1) implies by Proposition (2.2) that $G:=\operatorname{gr}_{A}(\mathfrak{p})$ is a domain and $G_{\mathfrak{m}}$ (and $A$ ) satisfy Serre's condition $S_{2}$. [M denotes the maximal homogeneous ideal of $G$ ].

Let $(y, z)$ be a minimal reduction of $p$. Since $\mu(p)=3$ by assumption, 
we find an element $\tilde{x} \in A$ such that $\mathfrak{p}=(\tilde{x}, y, z) A$, and $\tilde{x}^{2} \in(y, z) \mathfrak{p}$ by [HI1], Proposition 1.5. Hence:

$$
\tilde{x}^{2}+\tilde{B} \tilde{y} y+\tilde{C} \tilde{x} z+\tilde{D} y^{2}+\tilde{E} y z+\tilde{F} z^{2}=0
$$

for some $\tilde{B}, \cdots, \tilde{F} \in A$. Put $x:=\tilde{x}+(\tilde{B} / 2) y+(\tilde{C} / 2) z$. Then

$$
x^{2}+D^{\prime} y^{2}+E^{\prime} y z+F^{\prime} z^{2}=0,
$$

with some $D^{\prime}, E^{\prime}, F^{\prime} \in A$.

Since $A_{\mathfrak{p}}$ is regular, we have $\mathfrak{p} A_{\mathfrak{p}}=(y, z) A$ and therefore $x A_{\mathfrak{p}} \subset(y, z) A_{\mathfrak{p}}$, i.e. $\alpha x \in(y, z) A$ for some $\alpha \in A, \alpha \notin p$. Hence we find $\beta, \gamma \in \mathrm{A}$ such that $\alpha x+\beta y+\gamma z=0, \alpha \notin p$. Let

$$
\begin{aligned}
& K:=A / \mathfrak{p}, \quad R:=K[X, Y, Z] \text { and } \\
& \overline{\mathfrak{m}}:=\text { maximal ideal of } K .
\end{aligned}
$$

Clearly $\overline{\mathrm{m}} R$ is a prime ideal with $\mathrm{ht}(\overline{\mathrm{m}} R)=1$. Consider the natural epimorphism $\varphi$ :

$$
R \longrightarrow G=\operatorname{gr}_{A}(\mathfrak{p})
$$

Since $G$ is a domain by $(2.2), \operatorname{ker} \varphi=: I$ is prime in $R$ with $\operatorname{ht}(I)=1$. We denote the images of $D^{\prime}, E^{\prime}, F^{\prime}$ in $A / p$ by $D, E$ and $F$. Moreover we denote by $a, b, c$ the images of $\alpha, \beta, \gamma$ in $A / p$. Put

$$
\begin{aligned}
& f:=a X+b Y+c Z \text { and } \\
& g:=X^{2}+D Y^{2}+E Y Z+F Z^{2} \in K[X, Y, Z] .
\end{aligned}
$$

Then $f, g \in I$ and $f, g \neq 0$.

Claim 4.1. $(f): a^{2}=I$.

Proof. Since $R[1 / a] /(f) \cong K[1 / a][Y, Z]$ is a domain, $f R[1 / a]$ is a prime ideal. On the other hand, $I$ is a minimal prime ideal over $(f)$ and $a \notin I$. Therefore $f R[1 / a]=I R[1 / a]$. From this fact one can conclude that $f R=$ $I \cap Q$, where $Q$ is an $\overline{\mathfrak{m}} R$-primary ideal. Since $g \in I$ and $f R[1 / a]=I R[1 / a]$, $a^{n} g \in f R$ for some $n \in \mathbf{N}$, i.e.

(1) $a^{n} g=f u$,

where $u=u_{1} X+u_{2} Y+u_{3} Z, u_{i} \in K$. If $n \geqq 3$, comparing the coefficients on both sides of (1), we get $u_{1}, u_{2}, u_{3} \in a K$, hence $u \in a R$. Therefore (1) implies $a^{n-1} g \in f R$, so we may assume

(2) $a^{2} g \in f R$.

Since $g \notin \overline{\mathfrak{m}} R$ (and $Q$ is $\overline{\mathfrak{m}} R$-primary), $a^{2} \in Q$. Hence 


$$
(f): a^{2}=\left(I: a^{2}\right) \cap\left(Q: a^{2}\right)=I \cap R=I .
$$

Claim 4.2. $b^{2}, c^{2}, b c \in a^{2} K$.

Proof. By (2) in the proof of Claim (4.1) we have $a^{2} g=f \cdot u$ for some $u=u_{1} X+u_{2} Y+u_{3} Z \in R$. Comparing the coefficients on both sides of this equation we will obtain

$$
b^{2}+a^{2} D=0, \quad c^{2}+a^{2} E=0, \quad 2 b c+a^{2} F=0
$$

which proves the claim since $\frac{1}{2} \in A$.

Claim 4.2. $a K: b=a K: c$.

Proof. We will first show that $a K: c \subseteq a K: b$. If $d \in a K: c$, then

$$
a d b Y \in\left(f, a^{2}\right) R \subseteq\left(I, a^{2}\right) R .
$$

Note that $a^{2}, Y, Z \in R$ is a homogeneous s.o.p. on $G$. Moreover $G_{\mathrm{m}}$ satisfies Serre's condition $S_{2}$, and $G_{\Re}$ is a generalized Cohen-Macaulay ring by [HI2], Theorem 3.1. Therefore $a^{2}, Y$ is a regular sequence on $G_{\mathfrak{m}}$, hence on $G$ too. Using this fact we conclude from $(*)$ that

$$
a d b \in\left(I, a^{2}\right) R \cap K=a^{2} K,
$$

hence $d \in a K: b$.

By the symmetry between $b$ and $c$ we get $a K: b \subseteq a K: c$.

Clnim 4.4. $(a, c) K: b c a K: c$,

Proof. If $e \in(a, c) K: b$, then

$$
a c e Z^{2}=a e Z(f-a X-b Y) \in\left(f, a^{2}, a e b Y Z\right) R \subseteq\left(f, a^{2}, a c Y Z\right) R,
$$

where $a c Y Z=a Y(f-a X-b Y) \in\left(f, a^{2}, Y^{2}\right) R$. Hence

$$
\text { ace } \in\left(\left(I, a^{2}, Y^{2}\right) R: Z^{2}\right) \cap K=: S .
$$

To complete the proof, it is enough to show that $S=a^{2} K$ because then $c e \in a K$, i.e. $e \in a K: c$.

Since

$$
\begin{aligned}
S / a^{2} K & =\left(\left(Y^{2}, a^{2}, I\right) R: Z^{2} /\left(Y^{2}, a^{2}, I\right) R\right)_{0} \\
& =\left(0:_{G /\left(Y^{2}, a^{2}\right) G} Z^{2}\right)_{0} \subseteq H_{\mathfrak{M}}^{0}\left(G /\left(Y^{2}, a^{2}\right)\right)_{0},
\end{aligned}
$$

we need only to show that $H_{m}^{0}\left(G /\left(Y^{2}, a^{2}\right)\right)_{0}=0$. Since $a^{2}, Y^{2}$ is a regular sequence on $G$, we have the following exact sequences: 


$$
\begin{aligned}
& 0=H_{\mathfrak{M}}^{0}\left(G / Y^{2} G\right)_{0} \longrightarrow H_{\mathfrak{m}}^{0}\left(G /\left(a^{2}, Y^{2}\right) G\right)_{0} \longrightarrow H_{\mathfrak{M}}^{1}\left(G / Y^{2} G\right)_{0} \quad \text { and } \\
& H_{\mathfrak{M}}^{1}(G)_{0} \longrightarrow H_{\mathfrak{M}}^{1}\left(G / Y^{2} G\right)_{0} \longrightarrow H_{\mathfrak{M}}^{2}(G)_{-2} .
\end{aligned}
$$

By (2.1), $H_{\mathfrak{m}}^{1}(G)_{0}=0$ and $H_{\mathfrak{m}}^{2}(G)_{-2}=0$. Therefore, from the above exact sequences we obtain

$$
H_{\mathfrak{M}}^{0}\left(G /\left(a^{2}, Y^{2}\right) G\right)_{0} \subseteq H_{\mathfrak{M}}^{1}\left(G / Y^{2} G\right)_{0}=0 .
$$

This proves Claim 4.4.

§5. Proof of Theorem (1.2), (2) $\Rightarrow(1)$

Let $K$ be a local domain of dimension 1 and let $f=a X+b Y+c Z$ be an element in the polynomial ring $K[X, Y, Z]=: R$ with $a \neq 0$. We put

$$
\begin{aligned}
& G=R /(f): a^{2}, \quad M=\text { maximal homogeneous ideal of } R, \\
& H_{M}^{i}=\text { the } i \text {-th local cohomology functor on the category } \\
& \text { of graded } R \text {-modules. }
\end{aligned}
$$

By Theorem 2.1 we only need to prove:

$$
\begin{array}{ll}
H_{M}^{0}(G)=H_{M}^{1}(G)=0 \\
H_{M}^{2}(G)_{n}=0 \quad \text { for } n \neq-1 \\
H_{M}^{3}(G)_{n}=0 \quad \text { for } n \geqq 0 .
\end{array}
$$

Let $S$ denote the graded ring $R /\left(f, a^{2}\right) R$. Then there is the exact sequence

$$
0 \longrightarrow G \stackrel{\cdot a^{2}}{\longrightarrow} R / f R \longrightarrow S \longrightarrow 0 \text {. }
$$

Since $R / f R$ is a Cohen-Macaulay ring, we have

$$
H_{M}^{0}(G)=0, \quad H_{M}^{i+1}(G) \cong H_{M}^{i}(S)
$$

for $i=0,1$, where the isomorphism is of degree zero.

ClaIm 5.1. $H_{M}^{0}(S)=0$

Proof. We will show that $Y$ is a nonzerodivisor of $S$. Let $T$ be a form in $\left(a^{2}, f\right):_{R} Y$. We wil] denote by $\bar{K}$ the ring $K / a^{2} K$ and by $\bar{x}$ the image of an element $x$ of $K$ in $\bar{K}$. If $h$ is a polynomial in $R=K[X, Y, Z]$, we denote by $\bar{h}$ the corresponding polynomial in $\bar{K}[X, Y, Z]$. Then

$$
\bar{T} Y=\bar{H} \bar{f} \quad \text { for some form } H .
$$

Write $H=H_{1}+H_{2} Y$ with 


$$
H_{1}=a_{0} X^{n}+a_{1} X^{n-1} Z+\cdots+a_{n} Z^{n} \in K[X, Z] .
$$

Then $\left(\bar{T}-\bar{H}_{1} \bar{b}-\bar{H}_{2} \bar{f}\right) Y=\bar{H}_{1}(\bar{a} X+\bar{c} Z) \in \bar{K}[X, Z]$. Hence

$$
\bar{T}-\bar{H}_{1} \bar{b}-\bar{H}_{2} \bar{f}=\bar{H}_{1} \cdot(\bar{a} X+\bar{c} Z)=0 .
$$

Comparing the coefficients of this equation we get:

$$
\begin{aligned}
& \bar{a}_{0} \bar{a}=0 \\
& \bar{a}_{1} \bar{a}+\bar{a}_{0} \bar{c}=0 \\
& \vdots \\
& \bar{a}_{n} \bar{c}=0 .
\end{aligned}
$$

From the first $n$ equations we can deduce that there exist elements $b_{0}, \cdots, b_{n} \in K$ such that

$$
\begin{aligned}
& a_{0}=b_{0} a \\
& a_{1}=b_{1} a-b_{0} c \\
& \vdots \\
& a_{n}=b_{n} a-b_{n-1} c .
\end{aligned}
$$

Since $\bar{b}^{2}=\bar{c}^{2}=\bar{b} \bar{c}=0$ by assumption (2), (i) in Theorem (1.2) we get $\bar{b}_{n} \bar{a} \bar{c}=\bar{a}_{n} c=0$; hence $b_{n} \in a K: c=a K: b$ (by (2), (ii) in (1.2)) and therefore $\bar{b}_{n} \bar{a} \bar{b}=0$. Thus, using (*) and the fact that $\bar{a} \bar{b} X=\bar{b} \bar{f}$, we obtain $\bar{H}_{1} \bar{b} \in(\bar{f})$. Since $\bar{T}=\bar{H}_{1} \bar{b}+\bar{H}_{2} \bar{f}$, we get $\bar{T} \in(\bar{f})$, too, i.e. $T \in\left(a^{2}, f\right) R$. This proves Claim 5.1.

Claim 5.2. $\left[H_{M}^{1}(S)\right]_{n}=0$ for $n \neq-1$.

Proof. By the proof of Claim 5.1, $Y$ is a nonzerodivisor of $S$. Hence we have the exact sequence

$$
0 \longrightarrow S \stackrel{\cdot Y^{2}}{\longrightarrow} S \longrightarrow S / Y^{2} S \longrightarrow 0 \text {. }
$$

From this it follows that

$$
\left[O_{H_{M}^{1}(S)}: Y^{2}\right]_{n} \cong\left[H_{M}^{0}\left(S / Y^{2} S\right)\right]_{n+2}
$$

for all $n \geqq 0$. By the definition of local cohomology, $H_{M}^{0}\left(S / Y^{2} S\right)$ is submodule of the module $\bigcup_{t=1}^{\infty}\left(Y^{2} S: Z^{t}\right) / Y^{2} S$.

Assume that $\left[\bigcup_{t=1}^{\infty}\left(Y^{2} S: Z^{t}\right) / Y^{2} S\right]_{n}=0$ for $n \neq 1$. Then $\left[O_{H_{M S}^{1}(S)}: Y^{2}\right]_{n}$ $=0$ for $n \neq 1$. Hence $Y \cdot\left[O_{H_{M}^{1}(S)}: Y^{2}\right]=0$ or equivalently

$$
O_{H_{M}^{1}(S)}: Y=O_{H_{M}^{1}(S)}: Y^{2} .
$$

From this we can deduce that 


$$
O_{H_{M}^{1}(S)}: Y^{2}=O_{H_{M}^{1}(S)}: Y^{3}=\cdots=\bigcup_{t=1}^{\infty}\left(O_{H_{M}^{1}(S)}: Y^{\iota}\right)=H_{M}^{1}(S) .
$$

Hence from (3) we get for all $n \neq-1$

$$
\left[H_{M}^{1}(S)\right]_{n}=\left[O_{H}^{1}(S): Y^{2}\right]_{n+2}=0 .
$$

Therefore, it suffices to show that for all $n \neq 1$

$$
\bigcup_{t=1}^{\infty}\left[Y^{2} S: Z^{t}\right]_{n}=\left[Y^{2} S\right]_{n} .
$$

We will first show that

$$
\left[Y^{2} S: Z^{2}\right]_{n}=\left[Y^{2} S\right]_{n}
$$

or equivalently

$$
\left[\left(f, a^{2}, Y^{2}\right) R: Z^{2}\right]_{n}=\left[\left(f, a^{2}, Y^{2}\right) R\right]_{n}
$$

for $n \neq 1$. Consider the case $n=0$. Let $d \in K$ be an element of $\left[\left(f, a^{2}, Y^{2}\right) R: Z^{2}\right]_{0}$. Then

$$
d Z^{2} \in a^{2}(X, Y, Z)^{2} R+\left(f, Y^{2}\right) R .
$$

Hence there exist elements $u, v, w \in K$ such that modulo the ideal $\left(a^{2} Y Z, a^{2} Z^{2}, Y^{2}, f\right) R$ we have:

$$
\begin{aligned}
d Z^{2} & \equiv a^{2}\left(u X^{2}+v X Y+w X Z\right) \\
& \equiv u(b Y+c Z)^{2}-a v Y(b Y+c Z)-a w Z(b Y+c Z) \\
& \equiv-a(v c+w b) Y Z-a w c Z^{2},
\end{aligned}
$$

because $(b Y+c Z)^{2} R \in a^{2}(Y, Z)^{2} R$ by assumption (2), (i) of (1.2). From this we obtain modulo the ideal $f R$ a quadratic relation in the variables $Y, Z$. Since $Y, Z$ form a regular sequence on the factor ring $R / f R$, all coefficients of this quadratic form must be zero. Therefore, we can conclude that

$$
\begin{aligned}
& d+a w c \in a^{2} K \\
& a(v c+w b) \in a^{2} K .
\end{aligned}
$$

We can now use assumption (2), (ii) of (1.2) to deduce that

$$
w \in(a, c) K: b=a K: c
$$

and therefore $d \in a^{2} K=\left[\left(f, a^{2}, Y^{2}\right) R\right]_{0}$.

Now consider the case $n \geqq 2$. Let $F$ be an arbitrary element of 
$\left[\left(f, a^{2}, Y^{2}\right) R: Z^{2}\right]_{n}$. Then

$$
F Z^{2} \in a^{2}(X, Y, Z)^{4} R+\left(f, Y^{2}\right) R .
$$

Using (2), (i) of (1.2) we can easily see that

$$
a^{2}(X, Y, Z)^{4} R \subseteq a^{2}\left(Y^{2}, Z^{2}\right) R+f R .
$$

Therefore, there exists a form $H$, such that

$$
\left(F-a^{2} H\right) Z^{2} \in\left(f, Y^{2}\right) R .
$$

Since $Y^{2}, Z^{2}$ form a regular sequence on $R / f R$,

$$
F-a^{2} H \in\left(f, Y^{2}\right) R .
$$

Hence $F \in\left(a^{2}, f, Y^{2}\right) R$, as required.

So we have proven that $\left[Y^{2} S: Z^{2}\right]_{n}=\left[Y^{2} S\right]_{n}$ for $n \neq 1$. Similarly as for $\left(O_{H_{M}^{1}(S)}: Y^{2}\right)$ above, we can deduce from this relation that $Y^{2} S: Z^{2}=$ $\bigcup_{t=1}^{\infty}\left(Y^{2} S: Z^{t}\right)$ and therefore $\left[\bigcup_{t=1}^{\infty}\left(Y^{2} S: Z^{t}\right)\right]_{n}=\left[Y^{2} S\right]_{n}$ for $n \neq 1$. The proof of Claim 5.2 is complete.

From (2), Claim 5.1 and Claim 5.2 we get $H_{M}^{1}(G)=0, H_{M}^{0}(G)=0$, and $H_{M}^{2}(G)=0$ for $n \neq-1$. Now we are going to prove that $\left[H_{M}^{3}(G)\right]_{n}=0$ for $n \geqq 0$.

We consider again the exact Sequence (1). Since $Y, Z$ is a regular sequence on $R /(f)$ and $Y$ is a nonzerodivisor on $S$, we can deduce that $Y, Z$ is a regular sequence on $G$. Hence there are the following exact sequences

$$
\begin{aligned}
& {\left[H_{M}^{2}(G / Y G)\right]_{n} \longrightarrow\left[H_{M}^{3}(G)\right]_{n-1} \stackrel{\cdot y}{\longrightarrow}\left[H_{M}^{3}(G)\right]_{n}} \\
& {\left[H_{M}^{1}(G /(Y, Z) G)\right]_{n} \longrightarrow\left[H_{M}^{2}(G / Y G)\right]_{n-1} \stackrel{\cdot Z}{\longrightarrow}\left[H_{M}^{2}(G / Y G)\right]_{n} .}
\end{aligned}
$$

By assumption (2), (i) of (1.2) there are $D, E, F \in K$ with

$$
f(a X-b Y-c Z)=a^{2}\left(X^{2}-D Y^{2}-E Z^{2}-F Y Z\right) .
$$

Therefore $[G /(Y, Z) G]_{n}=0$ for $n \geqq 2$. This implies by [HIO], Corollary (35.21):

$$
\left[H_{M}^{1}(G /(Y, Z) G)\right]_{n}=0 \quad \text { for } n \geqq 2 .
$$

Since $\left[H_{M}^{2}(G / Y G)\right]_{n}=0$ for $n$ large, from (5) we can deduce that $\left[H_{M}^{2}(G / Y G)\right]_{n}$ $=0$ for $n \geqq 1$. Hence from (4) we similarly obtain $\left[H_{M}^{3}(G)\right]_{n}=0$ for $n \geqq 0$.

This completes the proof of Theorem (1.2), (1) $\Rightarrow(2)$. 


\section{§6. Example}

First we show that condition (2) of Theorem (1.2) already implies that $\mathfrak{p}$ is equimultiple and $A_{\mathfrak{p}}$ is regular in the following sense:

Lemma (6.1) Let $K$ be a one-dimensional local domain, s.t. $\frac{1}{2} \in K$. Let $a \neq 0, b, c$ be non-units in $K$ which satisfy the following conditions:

(i) $b^{2}, c^{2}, b c \in a^{2} K$

(ii) $a K: b=a K: c=(a, c) K: b$.

Let $R$ be the polynomial ring $K[X, Y, Z]$ and put $G:=R /(f): a^{2}$, where $f=a X+b X+c Z$. Let $\mathfrak{M}$ denote the maximal homogeneous ideal of $G$. Assume that $A=G_{\mathfrak{n}}$ and $\mathfrak{p}=(X, Y, Z) G_{\mathfrak{m}}$. Then $A$ is a domain with $\operatorname{dim}(A)=3$ and $\mathfrak{p}$ is a height 2 equimultiple prime ideal of $A$ such that $A_{\mathfrak{p}}$ is regular and $R(\mathfrak{p})$ is Cohen-Macaulay.

Proof. We know from [T], Korollar 1.6, that

$$
f R=I \cap Q,
$$

where $I$ is a prime ideal such that $I \cap K=(0)$ and $Q$ is an $m R$-primary ideal, where $m$ is the maximal ideal of $K$. By (i) we know that

$$
f \cdot(a X-b Y-c Z)=a^{2} \cdot\left(X^{2}-D Y^{2}-E Z^{2}-F Y Z\right)
$$

for some elements $D, E, F \in K$. Therefore

$$
X^{2}-D Y^{2}-E Z^{2}-F Y Z \in(f): a^{2}=I \cap\left(Q: a^{2}\right) .
$$

Since $X^{2}-D Y^{2}-E Z^{2}-F Y Z$ does not belong to any $\mathrm{m} R$-primary ideal, we can deduce that $Q: a^{2}=R$. Hence $(f): a^{2}=I$. That means $A$ is a domain with $\operatorname{dim}(A)=3$. By (1) the prime ideal $\mathfrak{p}$ is equimultiple (of height two). Since $\mathfrak{p} A_{\mathfrak{p}}=(Y, Z) A_{\mathfrak{p}}, A_{\mathfrak{p}}$ is regular. Using the fact that $\operatorname{gr}_{A}(\mathfrak{p})$ is isomorphic to $G$ we get by Theorem (1.2) that $R(p)$ is Cohen-Macaulay.

According to the lemma, to find a counterexample to the conjecture mentioned in section 1 we only need to find a one-dimensional local domain $K$ which contains appropriate elements $a, b, c$ :

ExAmple (6.2). Let $K$ be the ring $k \llbracket t^{5}, t^{6}, t^{7}, t^{8}, t^{9} \rrbracket$, where $k$ is a field with $\operatorname{char}(k) \neq 2$. Put

$$
a:=t^{5}, \quad b:=t^{8}, \quad c:=t^{9}, \quad d:=t^{6}, \quad e:=t^{7} .
$$

Then we have: 
(a)

$$
b^{2}=a^{2} d, \quad c^{2}=a^{2} b, \quad b c=a^{2} e
$$

(b)

$$
a K: b=a K: c=(a, c) K: b=(a, b, c, d, e) K \text {. }
$$

Let $A, \mathfrak{m}$, and $\mathfrak{p}$ be defined as in Lemma (6.1). Using the computer system COCOA developed by G. Niesi and F. Giovani we obtain $A=$ $(K[X, Y, Z] / I)_{M}$, where $M$ is the maximal homogeneous ideal of $\left.K \mid X, Y, Z\right]$ and $I$ is generated by the polynomials

$$
\begin{array}{ll}
a X+b Y+c Z, & b X+a d Y+a c Z, \\
d X+b Y+c Z, & c X+a c Y+a b Z, \\
e X-a^{2} Y-a d Z, & X^{2}-d Y^{2}-Z e Y Z-b Z^{2} .
\end{array}
$$

By Lemma (6.1) we know the Rees ring $R(p)$ is a Cohen-Macaulay ring. But $A$ is not Cohen-Macaulay because $Y, Z, a$ form a s.o.p. on $A$ but not a regular sequence: $X A \subseteq(Y, Z) A: a, X A \not \subset(Y, Z) A$.

\section{REFERENCES}

[CST] N. T. Cuong, P. Schenzel, N. V. Trung, Verallgemeinerte Cohen-MacaulayModuln, Math. Nachr., 85 (1978), 57-73.

[HI1] M. Herrmann, S. Ikeda, Remarks on liftings of Cohen-Macaulay property, Nagoya Math. J., 92 (1983), 121-132.

[HI2] - , On the Gorenstein property of Rees algebras, Manuscripta math., 59 (1987), 471-490.

[HI0] M. Herrmann, S. Ikeda, U. Orbanz, Equimultiplicity and blowing up, (Springer, Berlin-Heidelberg-New York, 1988).

[HMV] M. Herrmann, B. Moonen, O. Villamayor, Ideals of linear type and some variants, Queen's papers in Pure and Appl. Math. Nr. 83. The Curves Seminar at Queen's, Vol. VI, 1989.

[I] S. Ikeda, On the Gorensteinness of Rees algebras over local rings, Nagoya Math. J., 102 (1986), 135-154.

[T] N. V. Trung, Über die Übertragung von Ringeigenschaften zwischen $R$ und R/(F), Math. Nachr., 92 (1979), 215-229.

[TI] N.V. Trung, S. Ikeda, When is the Rees algebra Cohen-Macaulay?, Comm. Alg., 17 (12) (1989), 2893-2922.

\section{Herrmann}

Mathematisches Institut

der Universität zu Köln

5000 Köln 41

Germany

J. Ribbe

Mathematisches Institut

der Universität zu Köln

5000 Köln 41

Germany 
N. V. Trung

Institute of Mathematics

Box 631, Bò Hô

Hanoi

Vietnam 